\title{
The Digital Integrated Stratigraphy Project (DISP)
}

\author{
Axel Munnecke, Bradley D. Cramer, David P. Boon, Ranyah Kharwat, Carlos L. Aiken \\ \& DAVID I. SCHOFIELD
}

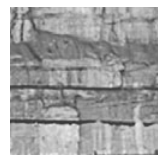

\begin{abstract}
The Digital Integrated Stratigraphy Project (DISP) aims to eliminate the stratigraphical ambiguity associated with sample position within a stratigraphical section. For example, it is often impractical or impossible to compare one author's measured section and data precisely against another author's geographically identical measured section and data due to discrepancies in the measured thicknesses of units, variations in the assignments of litho- and chronostratigraphical terms, and/or the precise line of measured sections between authors. The DISP will provide a solution to this problem by producing a precise 3-D digital rendering of the exposure using Terrestrial Laser Scanning (TLS) LiDAR, Real-Time Kinematic (RTK) Global Navigation Satellite Systems (GNSS), overlain digital photography, and GIS software to produce a $\mathrm{cm}$ to $\mathrm{mm}$-scale accurate digital version of a given exposure. Once available online as a permanent web-based digital resource for stratigraphy, researchers will be able to access the program and digital models of scanned exposures where they will have the means to log their sample positions directly onto the digital exposure while in the field, thus allowing unambiguous stratigraphical reference for future comparison. The Digital Integrated Stratigraphy Project represents the next generation of stratigraphical standard and can serve as the protocol for the future of high-resolution Palaeozoic Earth history research. • Key words: LiDAR, Palaeozoic, stratigraphy, high-resolution, Silurian, Telychian, GSSP.
\end{abstract}

Munnecke, A., Cramer, B.D., Boon, D.P., Kharwat, R., Aiken, C.L. \& SCHOFIELD, D.I. 2012. The Digital Integrated Stratigraphy Project (DISP). Bulletin of Geosciences 87(4), 705-712 (5 figures, 1 table, supplementary online material). Czech Geological Survey, Prague. ISSN 1214-1119. Manuscript received September 19, 2011; accepted in revised form May 30, 2012; published online July 19, 2012; issued October 17, 2012.

\begin{abstract}
Axel Munnecke (corresponding author), GeoZentrum Nordbayern, Fachgruppe Paläoumwelt, Universität Erlangen, Loewenichstraße 28, Erlangen D-91054, Germany; axel.munnecke@gzn.uni-erlangen.de •Bradley D. Cramer, Kansas Geological Survey/Department of Geology, University of Kansas, 1930 Constant Avenue, Lawrence, Kansas 66047, USA; current address: Department of Geoscience, University of Iowa, Iowa City, Iowa 52242, USA; cramerbd@gmail.com • David P. Boon, British Geological Survey, Kingsley Dunham Centre, Keyworth, Nottingham NG12 5GG, United Kingdom; dboon@bgs.ac.uk •Ranyah Kharwat \& Carlos L. Aiken, Cybermapping Laboratory/Department of Geosciences, ROC21, University of Texas at Dallas, 800 West Campbell Road, Richardson, Texas 75080-3021, USA; aiken@utdallas.edu • David I. Schofield, British Geological Survey, Columbus House, Green Meadow Springs, Tongwynlais, Cardiff CF15 7NE, United Kingdom; dis@bgs.ac.uk
\end{abstract}

The programs that began many decades ago to drill the ocean floor systematically (e.g. the Ocean Drilling Program or ODP) revolutionized Cenozoic and Mesozoic Earth history research by the very nature of the way the programs were conducted. Because there was a single core at each locality, from which each specialized researcher was expected to retrieve their data, a rigorous protocol was adopted by the shipboard scientific teams to ensure that all sampling endeavours on a given core would be carefully catalogued, cross referenced, and done in series. This guaranteed that there was little to no ambiguity associated with the direct comparison of multiple sets of data from multiple researchers from a given core, and has contributed to the proliferation of extremely high-resolution multi-proxy integrated Cenozoic and Mesozoic Earth history research over the past four decades.
The Palaeozoic community never had this experience, by virtue of the general lack of preserved Palaeozoic oceanic crust, and as a result there remains a critical roadblock to the potential resolving power of future Palaeozoic research. As a general rule, Palaeozoic Earth history research projects are conducted on a single to perhaps a few subjects at a time for any given section during any given field campaign. To accumulate multiple types of palaeontological, stratigraphical and geochemical data, all from one single Palaeozoic section, typically takes a matter of many years to sometimes more than a decade to produce during several field seasons. There is no a priori reason that it should take so long, but this is generally the pace when such objectives are not part of a globally organized research program. Over the course of the years required to amass from a single exposure palaeontological data from multiple clades, very 


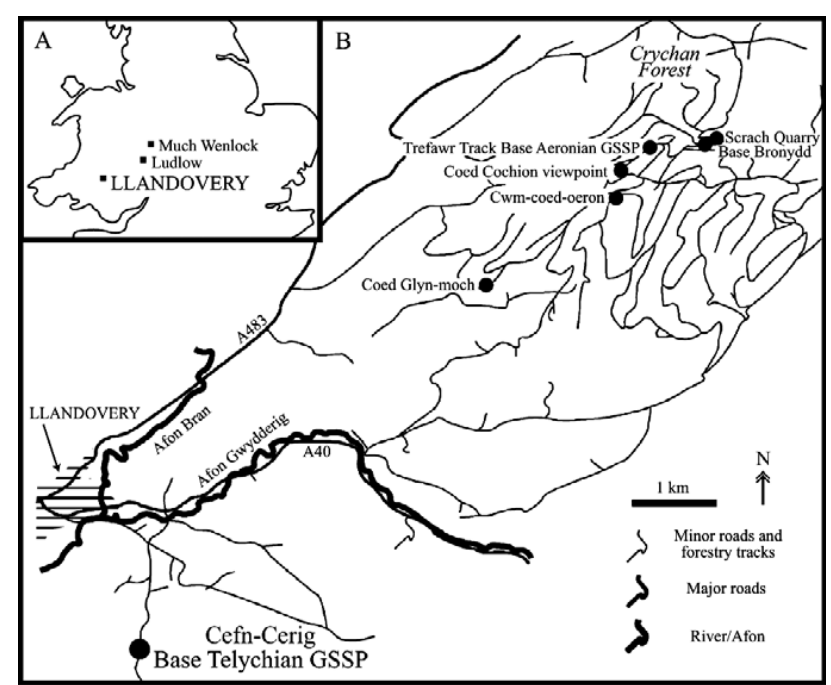

Figure 1. A - inset map of central England and Wales showing geographical places significant to the Silurian timescale. $\bullet B$ - map showing the town of Llandovery and important geological localities of the surrounding area. Both A and B modified from Davies et al. (2011).

high-resolution litho- and sequence stratigraphy, and a wide range of geochemical data, there is a necessary limit to the level of certainty with which each of the collected data sets can be compared. Because they were collected at different times and by different people, there is bound to be at least some degree of stratigraphical uncertainty associated with each sample. Highway paint and/or metal tags within exposures have been used to address this problem, but even they have a limit to their resolution.

Recent advances in geospatial data collection, analysis, and integration, such as the use of TLS (Terrestrial Laser Scanning) LiDAR combined with RTK (Real Time Kinematic) Global Navigation Satellite Systems (GNSS - Includes the U.S. GPS and Russian GLONASS satellites), high-resolution digital photography, and GIS software for three dimensional modeling of stratigraphical sections (e.g. Burnham et al. 2008, Kashouh et al. 2009, Aiken et al. 2009, Olariu et al. 2011), allow unique opportunities for the future of high-resolution stratigraphy and Earth history research (see http://www.utdallas.edu/research/interface). The capture of 3D exposures using LiDAR (Light Detection and Range), however, has mostly used point-clouds for analysis (McCaffrey et al. 2005), been utilized by reservoir modellers (Buckley et al. 2008, Fabuel-Perez et al. 2011), for geotechnical applications (Aiken et al. 2009), or geo-conservation (Woods et al. 2007), whereas the application of LiDAR mapping tools to global stratigraphical studies is in its infancy (Burnham et al. 2008, Kashouh et al. 2009). Here, we introduce the Digital Integrated Stratigraphy Project (DISP) as an interface between global stratigraphical studies for Earth history research and digital exposure mapping technology using LiDAR, and describe how we utilized terrestrial laser scanning (TLS) to make a high resolution, high accuracy, digital 3D photorealistic terrain model of a Palaeozoic Global Boundary Stratotype Section and Point (GSSP). The application of captured 3D exposures to Palaeozoic (and in fact all) global stratigraphical studies represents a unique opportunity to overcome previous practical limits to the potential resolving power of Palaeozoic research (i.e. stratigraphical ambiguity associated with sample position).

\section{Project architecture}

The Digital Integrated Stratigraphy Project lies at the intersection of a myriad of disciplines including palaeontology, litho-, bio-, and sequence stratigraphy, geochemistry and chemostratigraphy, structure and tectonics, geospatial and satellite technology, laser-based surveying and scanning technology, the internet, data-management and digital workspace innovation, and practical curatorial and library services. To be an effective web-based, open-access digital resource for stratigraphy, protocols will have to be developed for the integration of such varied data-sets into the DISP, and some of these details are discussed briefly below. The initial objectives of the development of the DISP were divided into two phases. Development Phase I includes the initial field acquisition and georeferencing of a few stratigraphical sections that are historically important to Palaeozoic Earth history research, as well as post-processing of the data for material demonstration of proof-of-concept (see below). Development Phase II will include development of the web-portal and user interface, as well as addressing long-term data management solutions.

\section{Development Phase I: field acquisition and post-processing}

Originally proposed as part of the International Geoscience Programme (IGCP) Project 591 - The Early to Middle Paleozoic Revolution, the Digital Integrated Stratigraphy Project was launched with an initial focus on early to middle Palaeozoic chronostratigraphy. To this end, the initial field acquisition of data took place during July 2011 when eight Silurian exposures were scanned and georeferenced prior to the Silurian Subcommission / IGCP 591 meeting in Ludlow, England. Four sections in Wales, including the base Aeronian and base Telychian GSSPs, and four sections in England, including the base Gorstian (base Ludlow) and base Ludfordian GSSPs, were chosen to begin Development Phase I of the DISP. Here, we have selected the base Telychian GSSP, in Cefn-Cerig Quarry (Fig. 1), to present as a material demonstration of digital stratigraphy. 


\section{Cefn-Cerig Quarry}

The Global Boundary Stratotype Section and Point (GSSP) 'golden spike' for the base of the Telychian Stage was placed in the Cefn-Cerig Quarry (U.K. Ordnance Survey Grid Co-ordinates SN 7743 3232), within bedded siliciclastics (muddy siltstones to sandstones) of the Wormwood Formation (Cocks et al. 1984). A level was chosen within a $29 \mathrm{~cm}$-thick bed below, which was a 'rottenstone' horizon that contained the Last Appearance Datum (LAD) of two key Aeronian brachiopod taxa, Stricklandia lens progressa and Eocelia intermedia (Cocks et al. 1984). Detailed remapping and new biostratigraphical data from the nearby Cefn-Cerig road section (including locality 163 of Cocks et al. 1984), have shown that the uppermost part of the Wormwood Formation in the nearby roadcut section (Fig. 2), above the uppermost strata in Cefn-Cerig Quarry, was truncated by a major intra-Silurian slide complex (Davies et al. 2010, 2011). The reader should consult Cocks et al. (1984) and Davies et al. (2010, 2011) for complete details of both the Cefn-Cerig Quarry and road sections.

\section{Field acquisition of data and georeferencing}

The LiDAR data was collected using a Riegl LPM-i800HA terrestrial laser scanner operated by Panasonic Toughbook running RiProfile software. The scanner has an $800 \mathrm{~m}$ range with accuracy of $\pm 15 \mathrm{~mm}$ and typically measures 1000 points per second using a near-infrared eyesafe laser (Laser Class 1). Each laser return defines a point on the rock face and the swath of points collected as the scanner rotates through a user-defined window yields a 'point cloud' which is the raw data consisting of several million points, each with an $x, y, z$ value and intensity value. A 12 megapixel coaxial camera with $85 \mathrm{~mm}$ lens is mounted on the scanner enabling the scanned object to be photographed. The colour value (RGB) is extracted from the images and superimposed on each point to produce a 'colour point cloud'. The photos can also be used to texture triangulated models to generate $3 \mathrm{D}$ photorealistic models.

The locations of the scans were recorded using differential GPS: we used a Leica Geosystems Viva CS15 controller with a Global Navigation Satellite Systems (GNSS) GS15 receiver. The dGPS unit receives Network RTK corrections from the SmartNet UK through an inbuilt GPRS (internet) mobile phone device. This dGPS configuration enables rapid positioning of the tripods to provide $3 \mathrm{D}$ accuracy down to $10 \mathrm{~mm}$, which minimizes time-consuming post-processing of dGPS data. The dGPS calculated position of each laser scan position is used in the processing stage to merge and register multiple scans and to perform referencing of the point cloud, to British National Grid pro-

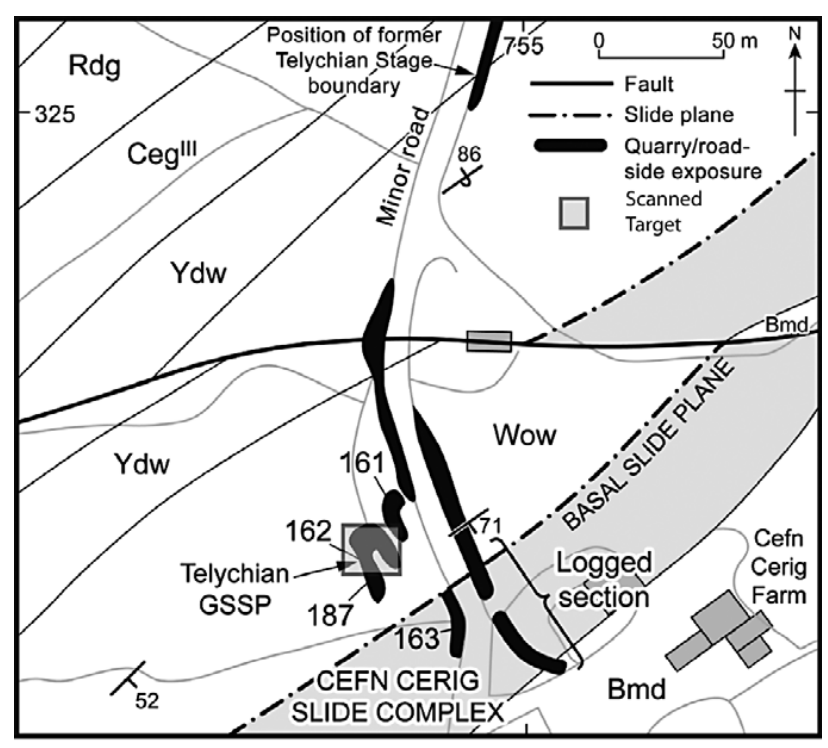

Figure 2. Locality map of Cefn-Cerig (Fron) Quarry and roadcut. Numbers refer to localities of Cocks et al. (1984). Original figure from Cocks et al. (1984) modified from Davies et al. (2011).

jection, allowing integration of the exposure model with other locally referenced data.

The scanning workflow involved setting up a pair of tripods along the length of the exposure. Light vegetation (moss, grass) covering the rock surface was cleared prior to the scanning. The scanner was set up on the first tripod and levelled, and the dGPS set up and set to log on the second tripod. Reflectors were placed on the surface to provide additional reference points common to both scans to assist draping of additional photographs to fill data gaps. At each tripod location a low-resolution wide-angled scan was first acquired to capture the general topography of the site, followed by a high resolution scan to capture detail of the GSSP. It was important that scans were acquired from two optimally spaced survey locations to ensure that the laser would have sufficient line-of-sight to characterize undulations on the rock surface. When the first scan was complete a reflector was mounted on the second tripod and fine-scanned to provide a known reference or 'tie' point and photographs were acquired. The laser scanner was then swapped onto the second tripod and the GPS swapped to the first tripod and the work flow repeated.

\section{Post-processing of data}

The University of Texas at Dallas (UTD) Cybermapping Laboratory has developed photorealistic 3D exposure modelling using surface modelling of 3D point clouds captured by Terrestrial Laser Scanners (Xu et al. 2000). The 3D point clouds are fitted with triangulated meshes upon 


\section{Creating 3D Photorealistic Model Flowchart}

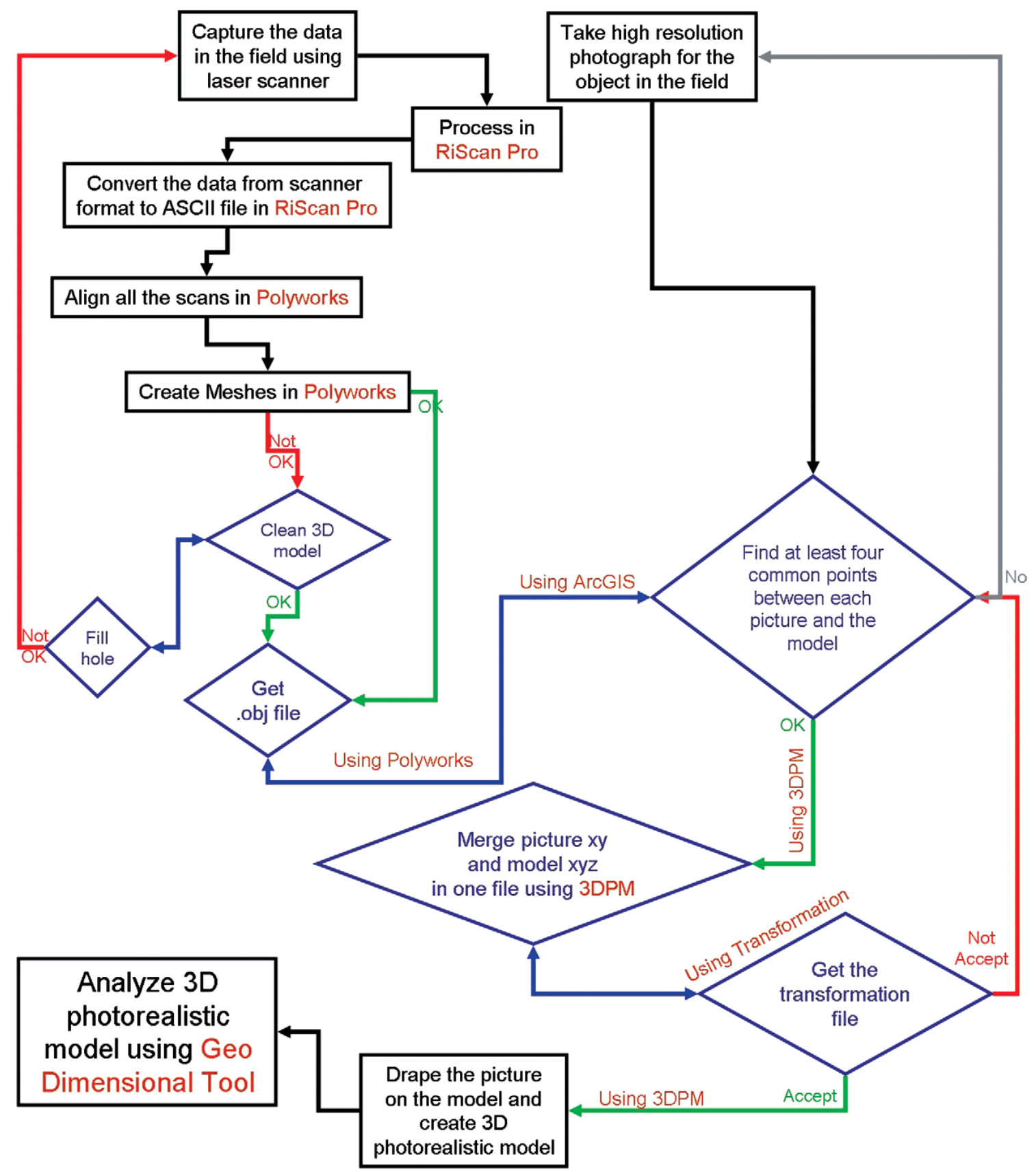

Figure 3. Work flow diagram for developing 3D photorealistic models.

which are draped digital photos, integrated and geo-referenced by accurate carrier phase satellite positioning, so that the surfaces and pixels of the imagery are located accurately and globally. As a result, each pixel (in RGB) now has spatial information - location ( $x, y$ and $z)$, intensity and, unlike the raw LiDAR data, colour as well.

The work flow and general procedure for three-dimensional (3D) photorealistic mapping with laser scanners de- veloped at the UTD Cybermapping laboratory were utilized for this investigation (Fig. 3). The exposure is scanned and global positioning system control is established. In the postprocessing of the data, the point cloud is edited, and then a triangulated mesh is created (and edited). Four common points are picked on both the model and the photos. Transformations relate the model to global coordinates and then the photo pixels are also transformed onto 


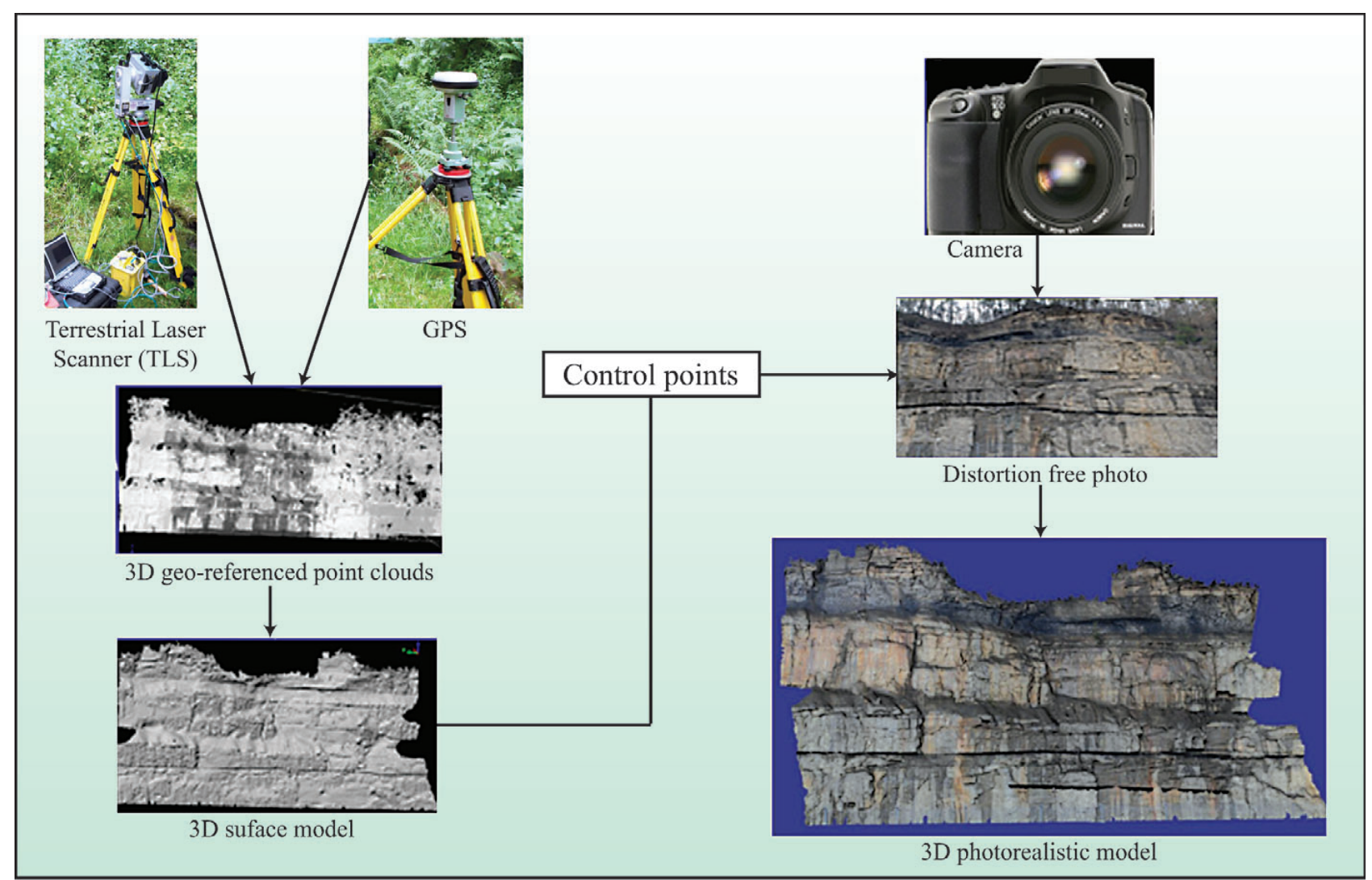

Figure 4. Summary diagram showing procedures for developing 3D photorealistic models (taken from Alfarhan et al. 2008).

the model to map (not draped), resulting in a 3D photorealistic model (from Alfarhan et al. 2008). A summarized version of the workflow for generating $3 \mathrm{D}$ photorealistic models of stratigraphical sections is shown in Fig. 4. In this case we applied GHVM GEOCONSTRUCTOR software that facilitates the processing by taking into account the many photographs taken by the attached coaxial camera (White et al. 2011).

Using these methods, a model of the Cefn-Cerig Quarry was built (Fig. 5A, B, Supplementary Online Material www.geology.cz/bulletin) with scans from sites $10 \mathrm{~m}$ apart and $16 \mathrm{~m}$ from the exposure (Table 1). The merged point cloud has 568,000 points and 151 photos were taken with the coaxial camera, finally using 26 photos in the model. The resulting model retains a realistic and accurate model of the actual exposure at a level determined by the equipment and processing that was applied (such as those discussed by Buckley et al. 2008). In the case presented here, the scanner and camera combination did not have the highest accuracy and resolution that could have been applied for this particular exposure using the latest generation of equipment, which could be potentially at sub millimetre. The objective of this work was not to demonstrate the highest resolution currently achievable, but rather, to demonstrate material proof of concept for Development Phase I of the DISP.
The photo colour information captured by the camera depends on the lighting at the time and the moisture content of the materials (e.g. grey mudstones and limestones generally appear darker when wet), and shadows do not contain useful colour information. So the colour of the model represents a possible "realistic" colouring after all the processing combining many photos taken over a period of time and perhaps any subsequent Photoshop-type massaging of the photos. The models can be viewed and interpreted on different software and hardware platforms (Xu et al. 2000, McCaffrey et al. 2005, Thurmond et al. 2005, Enge et al. 2007, Alfarhan et al. 2008, White et al. 2009, Olariu et al. 2010, Pringle et al. 2010). A 3D-pdf of the Cefn-Cerig Quarry is available as an online supplementary data file. This version of the model does not display the detail available in the original model due to the limitations of the $3 \mathrm{D}$-pdf venue but is meant to provide an easily accessible sample of the $3 \mathrm{D}$ model for material demonstration of

Table 1. GPS Locations (in British National Grid) of the two scan sites, Base Telychian GSSP (Cefn Cerig Quarry).

\begin{tabular}{lccccc}
\hline $\begin{array}{l}\text { Scanner } \\
\text { Position ID }\end{array}$ & $\begin{array}{c}\text { Height } \\
(\mathrm{m})\end{array}$ & $\mathrm{X}$ & $\mathrm{Y}$ & $\mathrm{Z}$ & $\begin{array}{c}\text { 3D CQ } \\
\text { Accuracy (m) }\end{array}$ \\
\hline SP1 & 1.82 & 77426.090 & 232342.415 & 181.540 & 0.061 \\
SP2 & 1.0652 & 77433.819 & 232335.830 & 180.156 & 0.023 \\
\hline
\end{tabular}




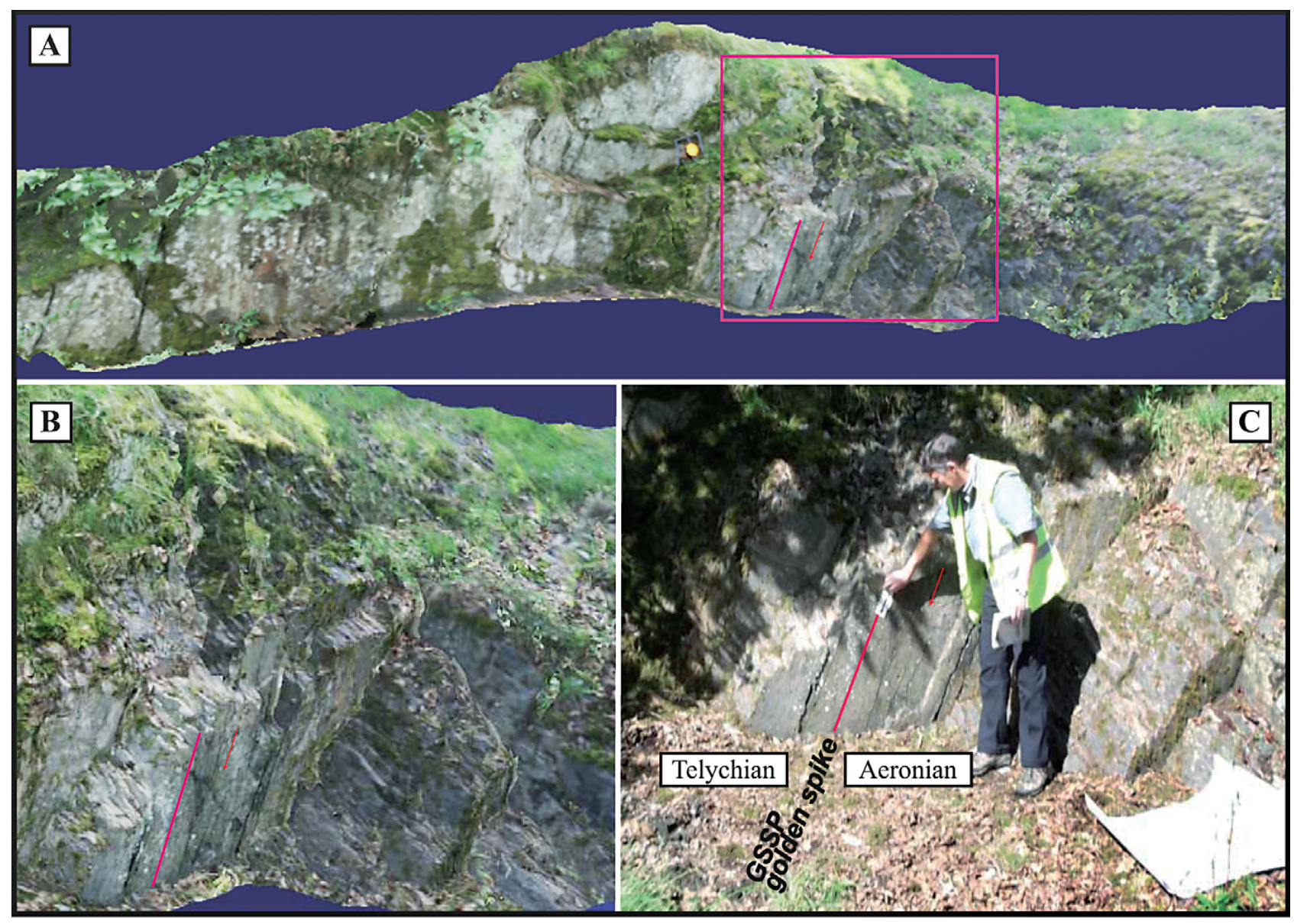

Figure 5. 3D Photorealistic Model (A, B) and photograph of the Cefn-Cerrig Quarry, GSSP for the base of the Telychian Stage. Red square in Panel A represents area shown in Panel B. Both are screen captures of the 3D pdf available as a supplementary online data file. The red line marks the GSSP 'golden spike'. The red arrow marks the position of the 'rottenstone' horizon containing the LAD of Stricklandia lens progressa and Eocelia intermedia. When viewing the 3D pdf, click to begin, left click to rotate, hold down both buttons to slide without rotation, right click and select 'full screen multimedia' for a full screen version. The vegetation is not defined in the triangulated mesh of the model because it is considered to be noise, so points capturing vegetation are extracted from the original point cloud, so that when the surface model, the triangulated mesh, is made, the vegetation appears as a green colour on the rock. The latest generation of scanners can produce significantly finer resolution than is depicted in the 3D pdf.

proof-of-concept. When this model is examined closely a patchwork of pieces of photographs can be seen because the GHVM GEOCONSTRUCTOR software that was used finds the pixels of the particular photo that are most perpendicular to the mesh triangular facets which then minimizes any smearing of the the pixels trying to fit a triangular facet, which results from using pixels from a less than optimized angle. A judicious use of Photoshop-type processing homogenizing the photos tones and hues can eliminate that appearance.

\section{Development Phase II: user interface and data management}

For the Digital Integrated Stratigraphy Project to be an effective web-based open-access digital resource for stratigraphy,
Development Phase II represents the critical next step. Once exposures are scanned, it will be necessary to enable end-users to access the captured exposures and log their sample positions and/or other notes directly onto the digital model while in the field. It is also critical that this information can then be uploaded back to the DISP website as a permanent repository of information available for future comparison. Currently in the initial design stage, Development Phase II has only just begun and a few initial notes are included below.

\section{Web-portal and user interface}

The entrance to the web-portal for the DISP will have two different paths into the system: one for scanner/developers, and the other for stratigraphical users. As no single group of researchers will be able to scan all of the sections that 
users may wish to have available, it is necessary that the DISP be able to accept captured exposures from a variety of TLS platforms and scanning parties. Therefore, appropriate protocols will be required to set standards for resolution and geo-referencing of captured exposures. Once a geo-referenced 3D photorealistic model of a captured exposure is uploaded and approved, it will then be made available to the stratigraphical user half of the DISP program.

The stratigraphical user will have the option of logging on to the DISP website directly from the field to access captured exposures, or of downloading a given exposure before leaving for the field (to allow for lack of internet coverage at the field locality). Using the technology demonstrated at http://www.utdallas.edu/igeology, the stratigraphical user interface will allow a series of information to be stored as separate files directly on the model. This information can then be uploaded back to the DISP website. For example, the user could upload a file containing sample positions, another file containing lithostratigraphical measurements and locations of lithostratigraphical boundaries determined by the user at the time of sampling, and another file with notes written directly on the exposure. Once uploaded back to the DISP website, the user could then wait to 'publish' their information as each file could be made available to other DISP users at a time chosen by the original stratigraphical user. Once the uploaded files have been made available to other users through the DISP system, anyone can then search for information from that exposure, and a series of files will show up as being attached to that exposure. The user could then choose their desired information to overlay against the model for future stratigraphical comparison.

\section{Long-term data management solutions}

The long-term utility of the Digital Integrated Stratigraphy Project will rest on our ability to find a permanent home and host for the online system. For the immediate future, demonstrations of the DISP will be housed at the IGCP 591 website (www.igcp591.org), however, a long-term data management solution is essential to the success of the project. It is in this capacity that the digital revolution intersects with practical curatorial and library sciences, and this component of the project is equally critical to its success. Further information and updates about Development Phase II will be posted at the DISP link on the IGCP 591 website as they become available.

\section{Conclusions}

The Digital Integrated Stratigraphy Project (DISP) is a tool to integrate virtually any type of geological/stratigraphical data into a visual, digital, cross-referenced system built upon 3D photorealistic models of exposures captured with Terrestrial Laser Scanning (TLS). The DISP will allow researchers to log their sample position and/or any other field notes directly onto the 3D model of the exposure, thereby providing unambiguous reference for future comparison. Presented as partial fulfillment of Development Phase I: Field Acquisition and Post-Processing; we have shown the processes required to produce a $3 \mathrm{D}$ photorealistic model of an exposure. The 3D pdf of the Cefn-Cerig Quarry (Supplementary Online Material) demonstrates the template upon which a stratigraphical user would begin logging their sample positions and field notes. The next step in the development of the DISP is to create the web-portal and user interface before finally securing a permanent home and host for the next generation of Digital Integrated Stratigraphy.

\section{Acknowledgements}

This work is a contribution to the International Geoscience Programme (IGCP) Project 591 - The Early to Middle Paleozoic Revolution, and was supported by grants from the German Research Council (DFG) to A.M. (Mu2352/2-1) and the National Science Foundation (NSF) to B.D.C. (EAR-0948277) and to C.L.A. (EAR-0632050, 0632102, 0651529, 0632402). David Boon and David Schofield publish with the permission of the Executive Director of the British Geological Survey. Lionel S. White Jr. provided the more efficient newly developed GEOCONSTRUCTOR software by Geological and Historical Virtual Models LLC, which was used in the photorealistic modeling. The authors thank Jeremy Davies and Richard Waters (both formerly of the BGS) for their logistical and technical assistance while scanning the Welsh Basin localities, as well as Mike Bevis and Peter Sadler for a detailed and insightful review that helped make this a stronger manuscript.

\section{References}

Aiken, C., Alfarhan, M., White, L. \& Abdelsalam, M. 2009. Three-dimensional globally-accessed monuments experiment (3D-GAME). The UT Dallas - Missouri S\&T Effort Joint Virtual Systems and Multi Media 2008 Conference Workshop Volume "Heritage in the Digital Era", Multi-Science Publishers, London, 119-129.

Alfarhan, M., Tuck, D., White, L. \& Aiken, C. 2008. Laser rangefinders and ArcGIS combined with three dimensional photorealistic modeling for mapping exposures in the Slick Hills, Oklahoma. Geosphere 4(3), 576-587. DOI 10.1130/GES00130.1

Buckley, S.J., Howell, J.A., Enge, H.D. \& Kurz, T.H. 2008. Terrestrial laser scanning in geology: data acquisition, processing and accuracy. Journal of the Geological Society, London 165, 625-638. DOI 10.1144/0016-76492007-100 
Burnham, B., Kashouh, M., Pujana, I., White, L., Alfarhan, M., BinolaR, A. \& AikEn, C. 2008. Three dimensional model of type section of Santonian limestone (Austin Chalk) at Ten Mile Creek, Dallas County, Texas: Applications to Paleontology of 3D Virtual Geologic Methods. Geological Society of America Joint Annual Meeting, 5-9 October, 2008, Houston, Texas.

Cocks, L.R.M., Woodcock, N.H., Rickards, R.B., Temple, J.T. \& Lane, P.D. 1984. The Llandovery Series of the type area. Bulletin of the British Museum (Natural History), Geology Series 38, 131-182.

Davies, J.R., Molyneux, S.G., Vandenbroucke, T.R.A., Verniers, J., Waters, R.A., Williams, M. \& Zalasiewicz, J.A. 2011. Pre-conference field trip to the Type Llandovery area, 29-72. In RAY, D.C. (ed.) Siluria Revisited: A Field Guide. International Subcommission on Silurian Stratigraphy, Field Meeting 2011.

Davies, J.R., Waters, R.A., Zalasiewicz, J.A., Molyneux, S.G., VAndenbroucke, T.R.A. \& Williams, M. 2010. A revised sedimentary and biostratigraphicalal architecture for the type Llandovery and Garth areas, Central Wales: a field guide. British Geological Survey Open Report OR/10/037.

Deng, H., Alfarhan, M., White, L.S., Oldow, J.S. \& Aiken, C. 2010. Reservoir characterization and flow simulation of a low-permeability gas reservoir: An integrated approach for modeling the Tommy Lakes Gas Field. 50(5), 32-47.

Fabuel-Perez, I., HodgetTs, D. \& RedFern, J. 2010. Integration of digital exposure models (DOMs) and high resolution sedimentology - workflow and implications for geological modelling: Oukaimeden Sandstone Formation, High Atlas (Morocco). Petroleum Geoscience 16, 133-154. DOI 10.1144/1354-079309-820

Kashouh, M., Burnham, B., Alfarhan, M., White, L., Pujana, I. \& Aiken, C. 2009. Site documentation of Global Stratotype Sections and Points (GSSPs) through methods of three dimensional geologic modeling - Case Study: The Wal-Mart Section of Ten Mile Creek, Dallas, Texas. Geological Society of America South-Central Section Meeting, 16-17 March, 2009, Dallas, Texas.

McCaffrey, K.J.W., Jones, R.R., Holdsworth, R.E., Wilson, R.W., Clegg, P., Imber, J., Holliman, N. \& Trinks, I. 2005. Unlocking the spatial dimension: Digital technologies and the future of geoscience fieldwork. Journal of the Geological Society (London) 162, 927-938. DOI 10.1144/0016-764905-017

Olariu, M.I., Aiken, C., Bhattacharya, J. \& Xu, X. 2011. Interpretation of channelized architecture using three-dimensional photo real models, Pennsylvanian deep-water deposits at Big Rock Quarry, Arkansas. Marine and Petroleum Geology 28(6), 1157-1170. DOI 10.1016/j.marpetgeo.2010.12.007

Olariu, C., Bhattacharya, J.P., Xu, X., Aiken, C.L.V., Zeng, X. \& McMechan, G.A. 2005. Integrated study of ancient delta front deposits, using exposure, ground penetrating radar and three dimension photorealistic data: Cretaceous Panther Tongue sandstone, Utah, 155-177. In Bhattacharya, J.P. \& GIOSAN, L. (eds) River deltas: concepts, models and examples. SEPM Special Publication 83.

Olariu, M.I., Ferguson, J.F., Aiken, C.L.V. \& Xu, X. 2008. Exposure fracture characterization using terrestrial laser scanners: Deep-water Jackfork sandstone at Big Rock Quarry, Arkansas. Geosphere 4(1), 247-259. DOI 10.1130/GES00139.1

White, L.S., Cline, J.R., Aiken, C., Alfarhan, M., Mills, G. \& Monsen, R. 2011. Rapid, high resolution, semi-automated system to capture 3D models of large geological exposures. SPAR LLC International Conference, Houston, TX.

Xu, X., Aiken, C. \& NeILsen, K.S. 1999. Real time and the virtual exposure improve geological field mapping. Transactions of the American Geophysical Union, EOS 80, 322-324.

Xu, X., Aiken, C., Bhattacharya, J.P., Corbeanu, R.M., NielSen, K.C., McMechan, G.A. \& Abdelsalam, M.G. 2000. Creating Virtual 3D Exposure. Leading Edge (Society of Exploration Geophysicists) February, 197-202. 